\title{
Aerodynamics of a Double-Element Wing in Ground Effect
}

\author{
Xin Zhang* and Jonathan Zerihan ${ }^{\dagger}$ \\ University of Southampton, Southampton, England SO17 1BJ, United Kingdom
}

\begin{abstract}
An investigation of a cambered, double-element, high-lift wing operating in ground effect was performed. The effect of ground proximity and flap setting was quantified in terms of aerodynamic performance and off-surface flowfield characteristics. From that, it was found that the flow is three-dimensional toward the wing tip with the main element generating most of the downforce but retains quasi-two-dimensional features near the center of the wing. However, at large heights the downforce increases asymptotically with a reduction in height. Then there is either a plateau, in the case of a low flap angle, or a reduction in downforce, in the case of a large flap angle. The downforce then increases again until it reaches a maximum and then reduces with decreasing height above the ground. The maximum downforce is dictated by gains in downforce from lower surface suction increases and losses in downforce caused by upper surface pressure and lower surface suction losses, with a reduction in height. For the high flap angle there is a sharp reduction just beyond the maximum, mainly because of the boundary layer separating, and a resultant loss of circulation on the main element.
\end{abstract}

\section{Nomenclature}

$b$

$C_{D}$

$C_{L}$

$C_{P}$

$c$

$c_{f}$

$h$

$q_{\infty}$

$R e$

$S$

$U_{\infty}$

$u, v, w=$

$u_{\min }$

$u^{\prime} u^{\prime}$

$x, y, z$

$\mu$

$\xi, \zeta$

$\rho_{\infty}$
$=$
$=$
$=$
$=$
$=$
$=$
$=$
$=$
$=$
$=$
$=$
$=$
$=$
$=$

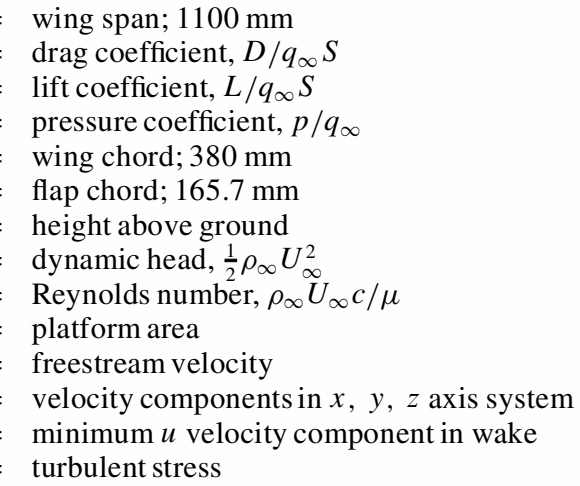

\section{Introduction}

A

WING operating in the proximity to the ground introduces different flow physics from that in freestream. Comparatively little information is placed in public domain about an inverted wing in ground effect, which has its applicationin the automobileindustry. For example, the front wing of a racing car operates in ground effect, at typical heights of $70-100 \mathrm{~mm}$ from the ground, ${ }^{1}$ and produces about $25-30 \%$ of the total downforce of the car. ${ }^{2}$ The downforce works in conjunction with the mechanical grip to improve the acceleration, braking, and cornering speed of the car. However, it is not only the overall level of downforce that is the important factor. The front wing changes height from the ground because of the suspension movements on the car. This severely affects the level

Received 4 April 2002; revision received 27 January 2003; accepted for publication 27 January 2003. Copyright (C) 2003 by Xin Zhang and Jonathan Zerihan. Published by the American Institute of Aeronautics and Astronautics, Inc., with permission. Copies of this paper may be made for personal or internal use, on condition that the copier pay the $\$ 10.00$ per-copy fee to the Copyright Clearance Center, Inc., 222 Rosewood Drive, Danvers, MA 01923; include the code 0001-1452/03 \$10.00 in correspondence with the CCC.

*Professor of Aerodynamics, Aeronautics and Astronautics, School of Engineering Sciences. Senior Member AIAA.

Research Student, Aeronautics and Astronautics, School of Engineering Sciences; currently Aerodynamicist, BAR Operation Centre, Brackley, England NN13 7BD, United Kingdom. of downforce, and hence the grip. It is important to maintain consistent levels of front end grip, not only for performance reasons. It is not only important to have a car that handles well for performance reasons, but it is also a significant safety issue. In addition to the aerodynamic performance of the front wing, another major issue is the wake/vortices that it generates. The flow to the undertray and diffuser in particular, but also the radiators and rear wing, is severely affected by the front wing because they all operate in the wake/vortices from the wing.

There has been a lack of data on inverted wings in ground effect that are supposed to simulate the flow correctly. Ground effect is normally felt within a height of one chord. Using a single-element wing, Zerihan and Zhang ${ }^{3}$ show that the use of a fixed ground would result in a substantial reduction in the downforce, with a significant drop below a critical height of around 0.3 chord (by as much as $25 \%$ ). Above the critical height major flow features would be the same as in the freestream case. Below the critical height, new features emerge, for example, shear-layer instability and wall jet, which will not be simulated correctly by the use of a fixed ground. Among various studies, Knowles et al. ${ }^{4}$ were the first to study experimentally a single-element wing with the suction surface near a moving ground. However, neither three-dimensional effects nor off-surface flowfield was studied. Recently, in a series of studies, Zerihan and Zhang ${ }^{3,5,6}$ conducted investigations of single-element wings in ground effect, including three-dimensionaleffect and offsurface flowfield surveys, as well as numerical modeling.

In practice, a typical geometry of the front wing is of a multielement configuration, which would introduce additional features of importance. The study of multielement flows is an area that has challenged researchers for a long time. Smith ${ }^{7}$ described the five beneficial effects of the gaps between the elements in multielement flows: slat effect, circulation effect, dumping effect, off-the-surface pressure recovery, and fresh-boundary-layer effect. Ranzenbach et al. ${ }^{8}$ demonstrated the ground effect for a double-element airfoil configuration. Their work begins to address the topic, using twodimensional model tests in a fixed ground wind tunnel on a NACA $63_{2}-215$ Mod B section with a $30 \%$ slotted flap for the doubleelement studies. Force reduction was observed. Jasinski and Selig ${ }^{9}$ presented an experimental study of a three-dimensional multielement wing in ground effect, again using a fixed ground facility. Two trailing vortices were observed rolling up from the end plate, the size of which increased for the larger flap deflection. In this study we aim to quantify the performance of a generic double-element wing in ground effect, employing model tests with correct ground conditions. Earlier results illustrate the large-scale unsteady and time-averaged flow features of a high-lift single-element wing. It shows that the ground has indeed a profound effect on the aerodynamic performance. By associating fluid flow measurements and 
observation with force and pressure measurements, it is possible to develop a greater understanding of flow physics.

\section{Methods}

\section{Test Facilities}

Tests were conducted in the University of Southampton $3.5 \times 2.5 \mathrm{~m}$ wind tunnel for laser Doppler anemometry (LDA) surveys (Fig. 1) and $2.1 \times 1.7 \mathrm{~m}$ wind tunnel for other tests. Details can be found in Ref. 10. Both tunnels are of a conventional closed-jet, closed-circuit design. At $30 \mathrm{~m} / \mathrm{s}$ the freestream turbulence is less than $0.2 \%$ in both tunnels. The tunnels are equipped with a large, moving belt rig.

\section{Wing Model}

Earlier studies were performed on a generic single-elementwing, with a cambered profile. ${ }^{3}$ Current tests employ the single-element wing as the main element in addition to a large flap. The main element has a modified General Aviation-Whitcomb (GAW) airfoil (Fig. 2). A detailed listing of the coordinates was given by Zerihan (see Table 3 in Ref. 10). The main changes include a forward movement of the lowest point on the suction surface, a flattening of the pressure surface, and a modification to the leading edge. The main purpose of the modification is to minimize the wake behind the airfoil. A span of $1100 \mathrm{~mm}$ was used, which corresponds to less than $75 \%$ of the width of the moving belt in the smaller wind tunnel, to minimize effects at the edge of the belt. The wing has a constant chord of $223.4 \mathrm{~mm}$.

A flap with a constant chord of $165.7 \mathrm{~mm}$ was used, the aft $35 \mathrm{~mm}$ of which is the thin region, $1 \mathrm{~mm}$ thick. A detailed listing of the flap coordinates was given in Ref. 10. Two flap angles were used (see the section on flap location optimization). For the high flap angle the chord is $378.9 \mathrm{~mm}$. For the lower flap angle the chord is $381.5 \mathrm{~mm}$. In presenting the results, all of the length scales were normalized by $380 \mathrm{~mm}$. The combined chord corresponds to an aspect ratio of 2.89 . End plates were employed, which are rectangular with dimensions $400 \times 170 \times 4 \mathrm{~mm}$ (Fig. 2).

\section{Tests}

All force, pressure, and flow visualizationtests were performed at a constant dynamic pressure of $56.25 \mathrm{~mm}$ water. LDA and particle

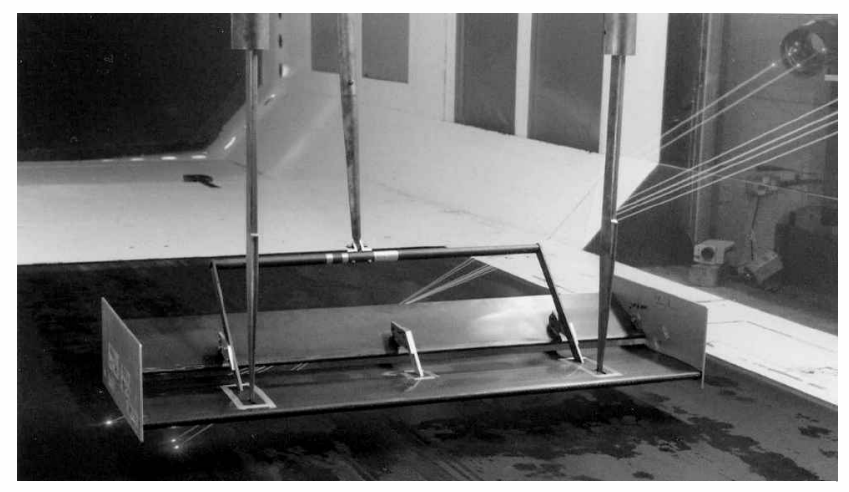

Fig. 1 Model installation in wind tunnel.

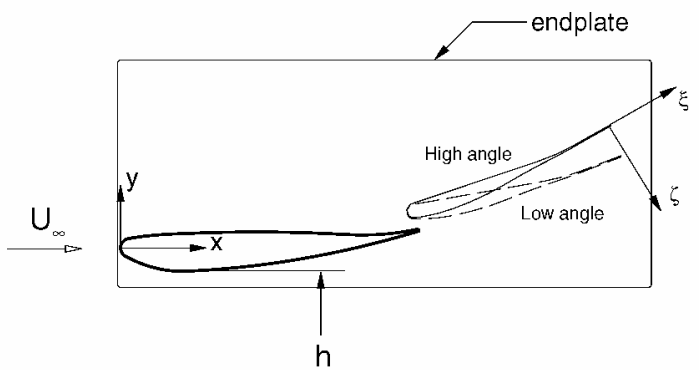

$\bigcup_{\infty}$ 1 moving ground

Fig. 2 Schematic of a double-element wing in ground effect. image velocimetry (PIV) tests were performed at a constant speed of $30 \mathrm{~m} / \mathrm{s}$. The Reynolds numbers were in the range $0.735 \times 10^{6}$ to $0.765 \times 10^{6}$ based on the total chord. The tests were performed transition free.

Two models were manufactured: a clean wing used for forces, flow visualization, LDA, and PIV; and an identical model, with the addition of pressure taps. The surface pressures were measured by a chordwise distribution of pressure taps, located near to the semispan. It comprises 25 taps on the suction surface and 23 on the pressure surface. A similar configuration was used for the 25 pressure taps on the flap. The chordwise group contains 13 taps on the suction surface and 12 taps on the pressure surface.

The forces and surface pressures acting on the wing have been measured for the model at a wide range of ground heights, from $1.97 c$ to less than $0.05 c$ above the ground. The height was defined by the distance from the ground to the lowest point on the wing with the wing incidenceset to 0 deg. The effect of changing the flap deflection angle was investigated at the different heights. The incidence of the wing was varied using a rotation about the quarter-chord position. The reference incidence of $1 \mathrm{deg}$ at which all double-element tests were performed is the incidence correspondingto end plates parallel to the ground, with the wing in its datum flap deflection, that is, a true incidence of $14.1 \mathrm{deg}$.

Off-surface results were also taken over a chordwise slice at the semispan of the wing with a LDA system to extract mean flow and turbulence data. LDA measurements were performed with a threecomponentDantec system with a 5-W argon-ionlasergenerator. The system was operated in backscatter mode. The velocities measured in the beam axes were resolved into the tunnel coordinate system $(x, y, z)$ using a matrix transformation. Seeding was introduced by three seeding generators located downstream of the rolling road, behind the model. The LDA signals were analyzed using three Dantec Burst Spectrum Analyzers. On average, a total of 800 bursts (instantaneous samples) were collected for each data point. The LDA study was supported by PIV survey of the flow between the flap and the ground, which provided insight into the state of the flow immediately behind the main element. PIV was performed using a Dantec PowerFlow system. The laser for the PIV system was located approximately $1.6 \mathrm{~m}$ downstream of the center of the wing, after the end of the rolling road. The region of the flowfield including the trailing-edge region, from the ground to above the flap extending to $x / c=1.2$ at the wing semispan, was mapped. The total number of samples recorded per run was 500 . The analysis sequence used was to cross correlate the data on $32 \times 32$ pixels and perform a range validation of the resulting vectors, generating a $157 \times 125$ grid. Details of the system can be found in Zerihan and Zhang. ${ }^{5}$ The range of heights extended from 22 to $100 \mathrm{~mm}$.

\section{Errors and Uncertainties}

The incidence of the wing was set to within $\pm 0.005 \mathrm{deg}$, and the height above ground was set to within $\pm 0.2 \mathrm{~mm}$. The constant dynamic pressure was set to 56.25 -mm water $\pm 0.05 \mathrm{~mm}$. The uncertainties in the force measurements were calculated using the addition method and a $95 \%$ confidence. ${ }^{11} C_{L}$ and $C_{D}$ have uncertainties of \pm 0.003 and \pm 0.0006 , respectively. Uncertainties in the surfacepressure results were calculated using the rss method as described by Moffat ${ }^{11}$ : the worst case corresponding to a $C_{p}$ of \pm 0.035 . The short-term repeatability was investigated; the highest uncertainties were found to be at the suction peak and the transition bubble, the worst corresponding to a $C_{p}$ of \pm 0.075 .

For the LDA survey the accuracy of the traverse is $0.01 \mathrm{~mm}$, but there is a gear backlash of $0.5 \mathrm{~mm}$. Attempts were made to reduce the effect of this by always approaching a boundary layer or wake profile from the same direction. Following an analysis by Zhang, ${ }^{12}$ an estimate of the uncertainty in the velocity measurement gives $u / U_{\infty} \pm 0.005$ and $v / U_{\infty} \pm 0.005$. An estimate of the $95 \%$ confidence level has been performed following procedures given by Benedict and Gould. ${ }^{13}$ In a typical test the worst uncertainty is 0.006 for $u^{\prime} u^{\prime} / U_{\infty}^{2}=0.007$, or less than $10 \%$ of the value. For the $u$ velocity the worst uncertainty is less than $0.1 \%$. 


\section{Results and Discussion}

\section{Flap Location Optimization}

For the forces two flap angles were used: the datum (high flap angle) and a deflection of $-8.5 \mathrm{deg}$ (low flap angle). The point about which the flap was rotated was at a location of $x / c=0.567$, $y / c=0.076$, that is, $2 \mathrm{~mm}$ downstream from the leading edge of the flap (see Tables 3 and 4 in Ref. 10 for the exact coordinates).

A series of tests were performed in order to find the flap location at which the maximum downforce was produced for a constant flap deflection. The gap and the overlap were varied in steps of $2 \mathrm{~mm}$. The overlap was defined as the horizontal distance between the trailing edge of the main element and the leading edge of the flap, with a positive overlap for the flap leading edge upstream of the mainelement trailing edge. The gap was defined as the vertical distance between the trailing edge of the main element and the lowest point on the flap suction surface, with a positive gap for the flap leading edge above the main-element trailing edge. The flap location optimization was performed for the datum flap deflection angle at an arbitrary height of $h / c=0.263$. Results of the optimization can be seen in Fig. 3. The optimum location for the flap can be seen to be an overlap of $0.024 c$ and a gap of $0.032 c$. These correspond to 9 and $12 \mathrm{~mm}$, respectively. This was used as the location of the flap for all further tests at the different heights and flap deflection.

\section{Oil Flow Visualization}

Oil flow visualization was performed at various heights (see examples in Fig. 4). Although it difficult to see from the pictures, the streaklines on both elements featured spanwise components, particularly near the tips. It seems, however, that over the center the surface streaklines do not feature significant spanwise components. The flow over the central portion can be regarded as quasi-twodimensional. Significant three-dimensional effect is observed near the tip, which is probably associated with the likely presence of the edge vortices. In fact a recent single-element wing study suggests that the breakdown of the edge vortices causes the change in the lift slope between regions a and $\mathrm{b}$ (see later) ${ }^{14}$ It is conceivable that the pressure field could be affected near the center. (Further studies need to be performed on this.) Nevertheless, the main tenet of the current paper remains valid.

At $h / c=0.395$ (Fig. 4a) the separation bubble indicating transition is clear over most of the span of the wing. The separation point in the bubble was measured at $x / c=0.11-0.13$, with turbulent reattachment at $x / c=0.16$. However, near the center of the wing, a small region can be seen where the bubble is breaking up just to the left of the semispan and where the bubble is not present slightly to the right of the semispan. Close inspection of the patterns on the wing, difficult to see in the figure, reveals a small bubble very close to the leading edge, $x / c=0.01-0.02$, where there is no main transition bubble. (This phenomenon is discussed further in the following paragraph.) Hence, on the main element, transition is observed at two chordwise locations at this height for the low flap angle: $x / c=0.01-0.02$ for a small region at the center of the

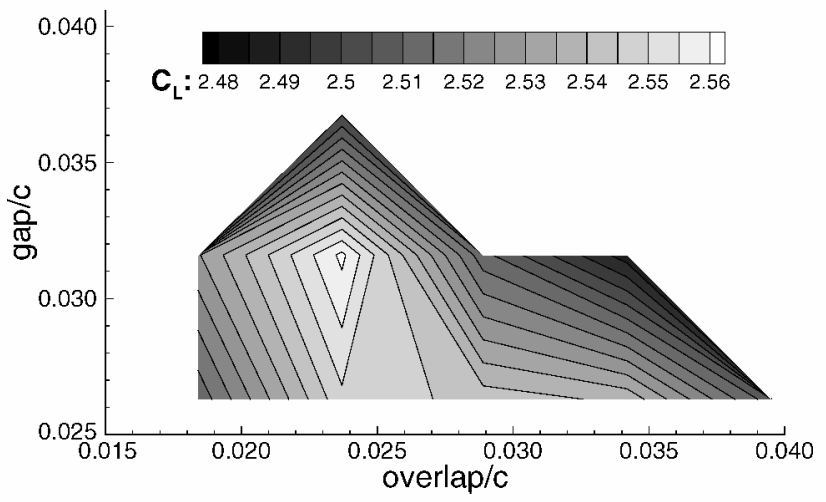

Fig. 3 Effect of varying overlap and gap at $h / c=0.263$; datum flap angle.

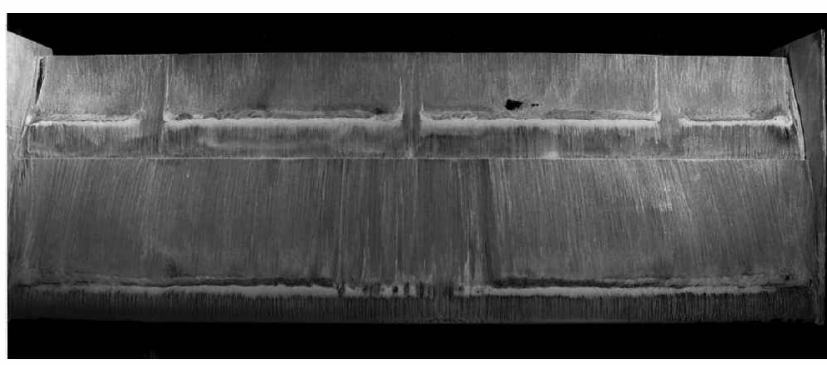

a) $h / c=0.395$, low flap angle

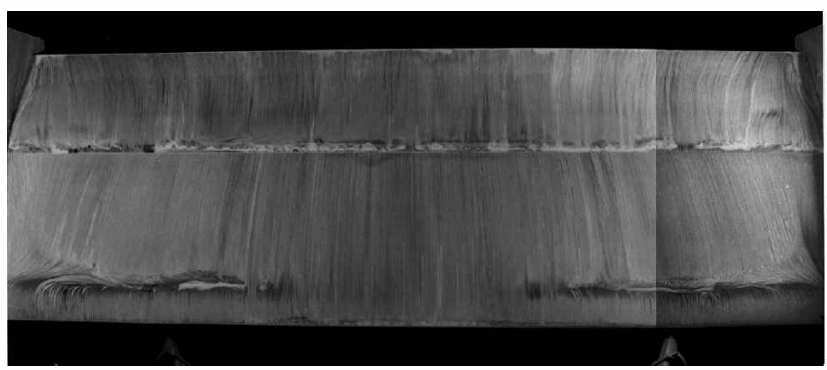

b) $h / c=0.211$, high flap angle

Fig. 4 Oil flow visualization on suction surface showing leading-edge lowermost.

wing and $x / c=0.11-0.16$ elsewhere. On the flap a reasonablylarge transition bubble is again seen. It is broken up at three spanwise locations where the brackets secure the flap in place. The separation point was measured at $x / c=0.69-0.72$, with turbulent reattachment at $x / c=0.75$. The leading-edge transition was not seen at $h / c=0.211$, and heights below this, for the low flap angle. On the main element the detachment point in the bubble was measured at $x / c=0.12-0.14$, with reattachment at $x / c=0.17$, that is, at approximately the same location within the measurement uncertainty The bubble on the flap was measured at the same position as that at $h / c=0.395$.

For the high flap angle there is a significant difference (Fig. 4b). On the main element transition at the leading edge accounts for a significant portion of the span of the wing. In the central portion of the image, the leading-edge bubble can be seen, and on the right the bubble is farther back, at $x / c=0.11-0.18$. As at the low flap angle, this portion of the wing with leading-edge transition reduces as the ground height is reduced. At $h / c=0.211$ it is approximately $36 \%$ of the span. This reduces from $57 \%$ at $h / c=0.395$ to $46 \%$ at $h / c=0.263,36 \%$ at $h / c=0.211,20 \%$ at $h / c=0.158$, and to virtually zero at $h / c=0.105$. The transition location for the flap is now very close to the leading edge, at $x / c \approx 0.58$. This was found to be the case for all heights tested for the high flap angle.

\section{Force Behaviors}

The downforce and drag coefficients as the ride height is varied are given in Figs. 5a and 5b. It can be seen that the basic characteristics of the downforce with height curve are broadly similar to the single-element wing. ${ }^{3}$ The downforce increases as the height reduces, and eventually a maximum downforce is obtained, after which the downforce reduces sharply. For the low flap angle the maximum is reached at $h / c=0.066$, and for the high flap angle $h / c=0.079$. Below the maximum downforce height is the downforce reduction region $c$.

However, for the low flap angle at a height of $h / c=0.171$ a discontinuity in slope can be seen as the trend of increasing downforce with height reduction abruptly stoppings; just above this height the gradient of the line is high, and just below this point the gradient is low. As the height is reduced further, the slope of the line increases again, in a similar manner to that at large heights, until it gradually reduces, and the downforce eventually reaches the maximum, corresponding to $C_{L}=2.588$. To aid in describing the aerodynamic characteristics, heights greater than and equal to $h / c=0.171$ 


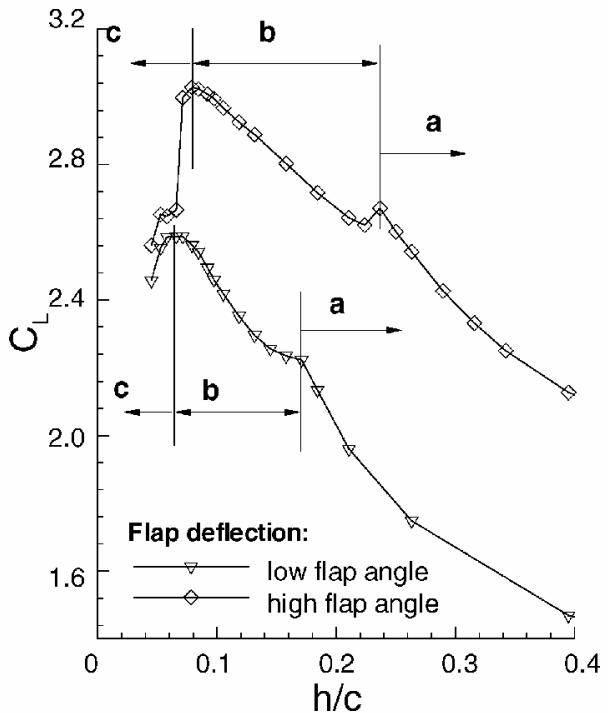

Fig. 5a Downforce with ground height.

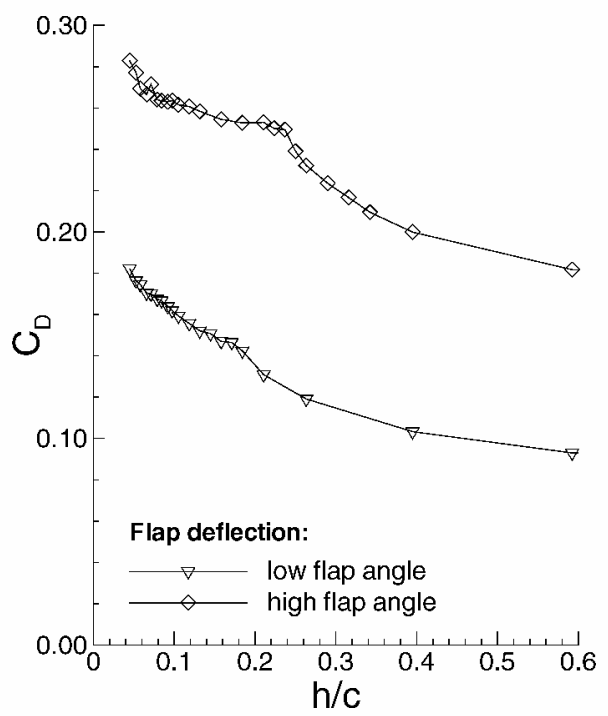

Fig. 5b Drag with ground height.

for the low angle flap angle will be referred to as region $a$, and heights between $h / c=0.066$ and $h / c=0.171$ will be referred to as region $b$.

For the high flap angle the wing generates significantly more downforce than for the lower flap angle. At large heights, greater than $h / c=0.237$, similar characteristics can again be seen as the downforce increases asymptotically as the height is reduced. However, a discontinuity in slope again exists in the curve, at $h / c=0.237$, and the downforce actually reduces suddenly just below this height. As the height is reduced further, the portion of the curve seems more linear. A maximum in the downforce occurs at $h / c=0.079$, corresponding to $C_{L}=3.028$. Below this height a sudden drop in the downforce is observed. In a similar manner to the low flap deflection, three flow regions are defined.

The variation of drag with height is given in Fig. 5b. The curves for the two flap deflections show a general trend of increasing drag as the height is reduced. At the low flap deflection the curve is relatively smooth, with the gradient of the line generally increasing as the ground height is reduced. However, just above $h / c=0.171$, at the lower boundary of the type $a$ flows at this flap deflection, the gradient is greater than just below this height, that is, a similar characteristic to the downforce against height curve is observed. At the high flap deflection a significantly greater drag is obtained at all heights, than compared to the low flap deflection. A broadly similar variation with height is observed. In the border between the type

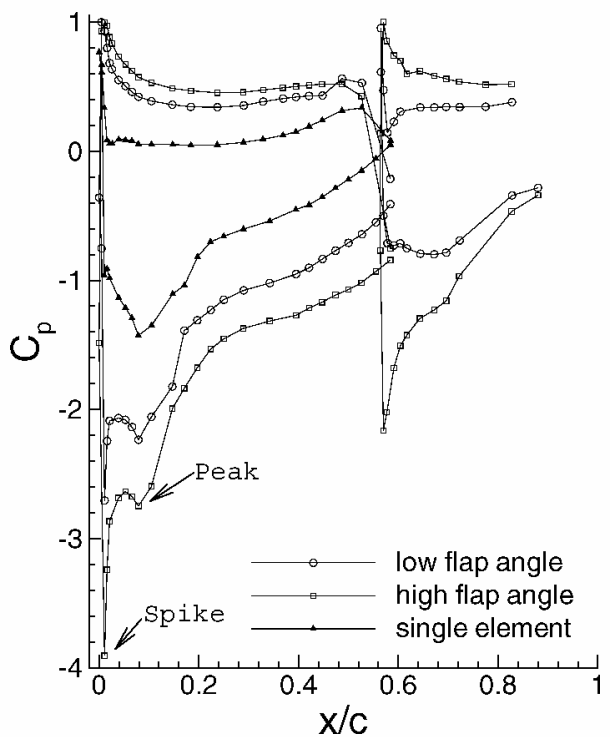

Fig. 6 Chordwise surface pressures at wing center at $h / c=1.97$.

$a / b$ flows, at $h / c=0.237$, the general trend is for a sharper gradient at low type $a$ heights, and a lower gradient at greater type $b$ heights. This is somewhat more pronounced than for the low flap deflection.

\section{Chordwise Pressure Distribution}

A comparison of pressure distributionat $h / c=1.97$ is made of the single-element wing and double-element wing (Fig. 6). The flap has the effect of introducing a finite pressure over the trailing edge of the main element. The pressures on the pressure surface are increased, and the suction generated on the suction surface also increases compared to the single-elementwing. The increments are greater for the high flap angle than for the low flap angle. Over the mid and aft portions of the wing, the increases are broadly constant for both flap angles. However, to $x / c \approx 0.15$ the shape of the distribution changes more significantly for the double-element wing compared to the single-element wing. On the pressure surface the acceleration of the flow from stagnation at the leading edge is smoother with the flap, in the region to $x / c \approx 0.1$. For the single-element wing the flow reaches a velocity approaching freestream at $x / c \approx 0.02$. On the suction surface, the suction peak for the single-element wing at $x / c=0.08$ remains at the same place. This will be known as the suction peak. However, a suction spike near to the leading edge at $x / c=0.01-0.02$ becomes apparent when the flap is added, which shall be referred to as the suction spike. The spike grows for the higher flap angle. The bump that is present in pressure on the singleelement suction surface at $x / c=0.17$ representing the transition bubble is not as apparent for the double-element wing.

The two flap angles yield different types of distributions. For the low flap angle the flow is accelerated over the suction surface a little from the higher than freestream velocity near to the main element trailing edge. It remains at $C_{P} \approx-1$ until $x / c=0.72$, and then the recovery starts as the trailing edge is approached. For the high flap angle the flow is accelerated rapidly from the leading edge at $x / c=0.564$ to a peak suction at $x / c=0.570$. The flow is then retarded. The difference in suction between the high and low flap angles reducesalong the chord of the flap and is small for the final tap at $x / c=0.880$. On the pressure surface the flow is accelerated from stagnation at a greater rate for the low flap angle. The pressures remain broadly constant for each configuration but are greater in magnitude for the high flap angle than for the low angle.

The chordwise $C_{P}$ distributions for the low flap angle are presented in Fig. 7. For the large heights (Fig. 7a), when the ride height is reduced, the suction increases on the lower surface of both the main element and the flap. The increase, however, appears greater on the main element when compared to the flap. The suction near the trailing edge of the main element increases with increasing ground proximity, the magnitude of which is comparable to the increase in 


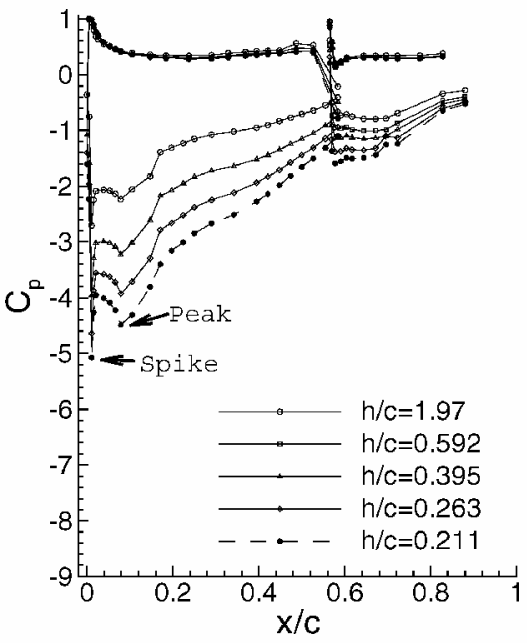

a)

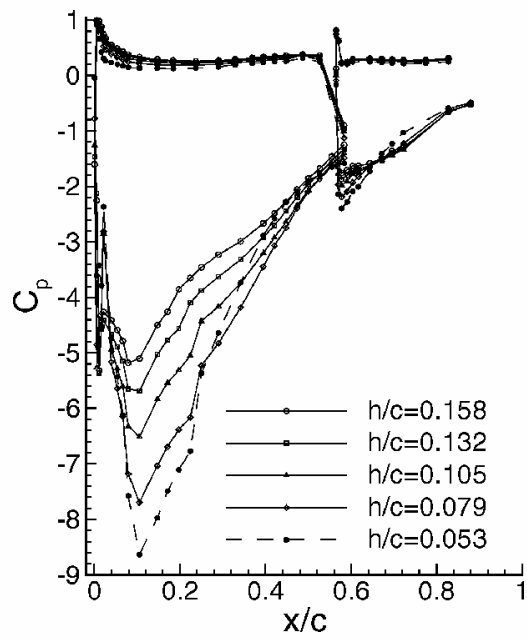

b)

Fig. 7 Chordwise surface pressures at wing center for low flap angle.

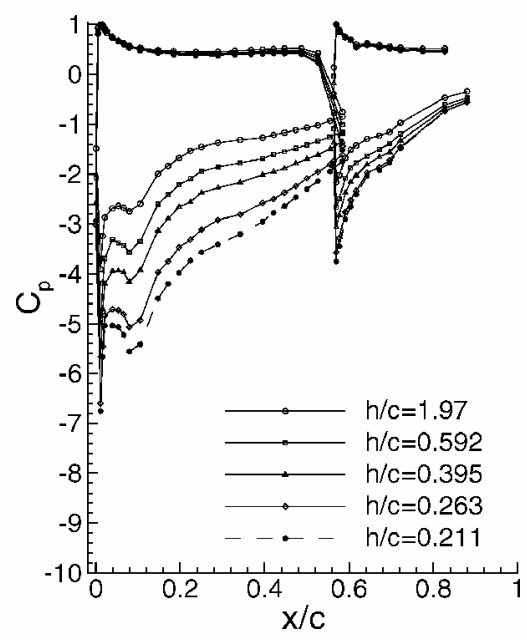

a)

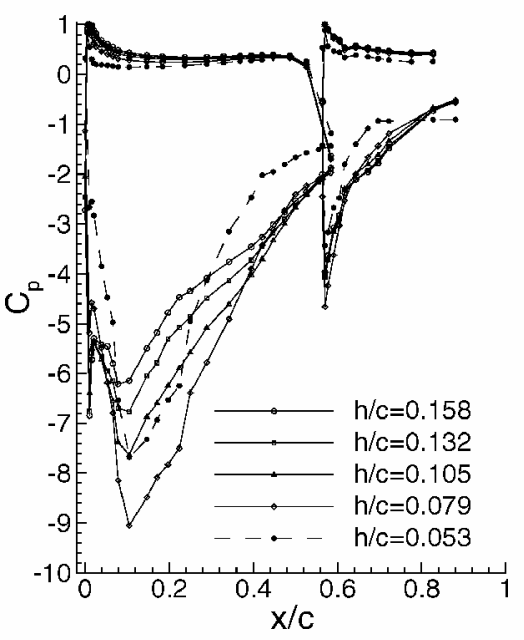

b)

Fig. 8 Chordwise surface pressures at wing center for high flap angle.

suction near the flap leading edge. The fundamental shape of the distributions does not change, and the main suction peak, and the spike very close to the leading edge, are still present as the ground is approached and remain at the same location within the spatial resolution of the taps. The reduction in pressure from after the spike, to the main peak increases as the height is reduced. The distribution over the pressure surface varies little from $h / c=1.97$ to 0.211 .

Closer to the ground (Fig. 7b), the effect differs. The suction on the main element increases significantly as the height is reduced, especially in the chordwise region from $x / c=0.08$ and downstream. The increment starts to reduce from $x / c \approx 0.25$ and is small at the trailing edge of the main element. For the lowest height, $h / c=0.053$, a reduction in suction is found from $x / c=0.25 \mathrm{com}$ pared to $h / c=0.079$. The tap recording the suction peak moves from $x / c=0.079$ to 0.105 as the height is reduced. The characteristics of the spike near to the leading edge also change. Although not overly clear, at heights lower than and including $h / c=0.132$ the maximum suction over the entire surface is found in the suction peak at $x / c=0.105$, compared to the leading-edge spike at heights greater than this. The peak suction at the lowest height corresponds to $C_{P}=-8.7$, much greater than the maximum for the single-element wing. Over the lower surface of the flap, the change in suction is small compared to the main element. There is a tendency for the suction to increase near to the leading edge, with the lowest height case showing a slight reduction in suction from $x / c=0.65$. The distributions over the pressure surface of both elements changes very little, but the slight effect of reducing pressure with height can be seen.
Results for the high flap angle (Fig. 8) show a similar effect of the ground on the pressure distributions. At the large heights (Fig. 8a) there is a large increase in suction on the main element lower surface as the height is reduced. Over the flap lower surface the increase in suction is not as significant and is very small for $h / c=0.211$. Again, the suction spike very near to the leading edge of the main element provides the greatest overall suction at these heights. $C_{P}$ distribution on the pressure surface experiences little variation as the height is reduced. At the lower heights (Fig. 8b), for all heights apart from the lowest of $h / c=0.053$, the lower surface pressures on the main element reduce, most significantly over the region from $x / c=0.1$ to 0.25 . The suction peak close to this chordwise position moves aft, from $x / c=0.079$ to 0.105 with the reduction in height. Again, this suction peak contains the maximum suction over the entire wing for all heights lower than and including $h / c=0.132$. At greater heights than this, the maximum overall suction is found in the suction spike at the leading edge. For the flow over the flap suction surface at all heights apart from the lowest, the suction changes very little as the height is reduced in this height range. There is a slight tendency for increasing suction in the peak upstream of $x / c=0.63$ and reducing suction downstream of this, as the height is reduced. For the lowest height of $h / c=0.053$, the suction over the main element lower surface reduces significantly over the entire surface. A constant pressure region is observed on the flap from $x / c=0.7$, also with reduced suctions on the flap upstream of this. For both the main element and the flap, the pressure reduces very slightly over the upper surface with a reduction in height. An additional reduction is observed at the lowest height. 

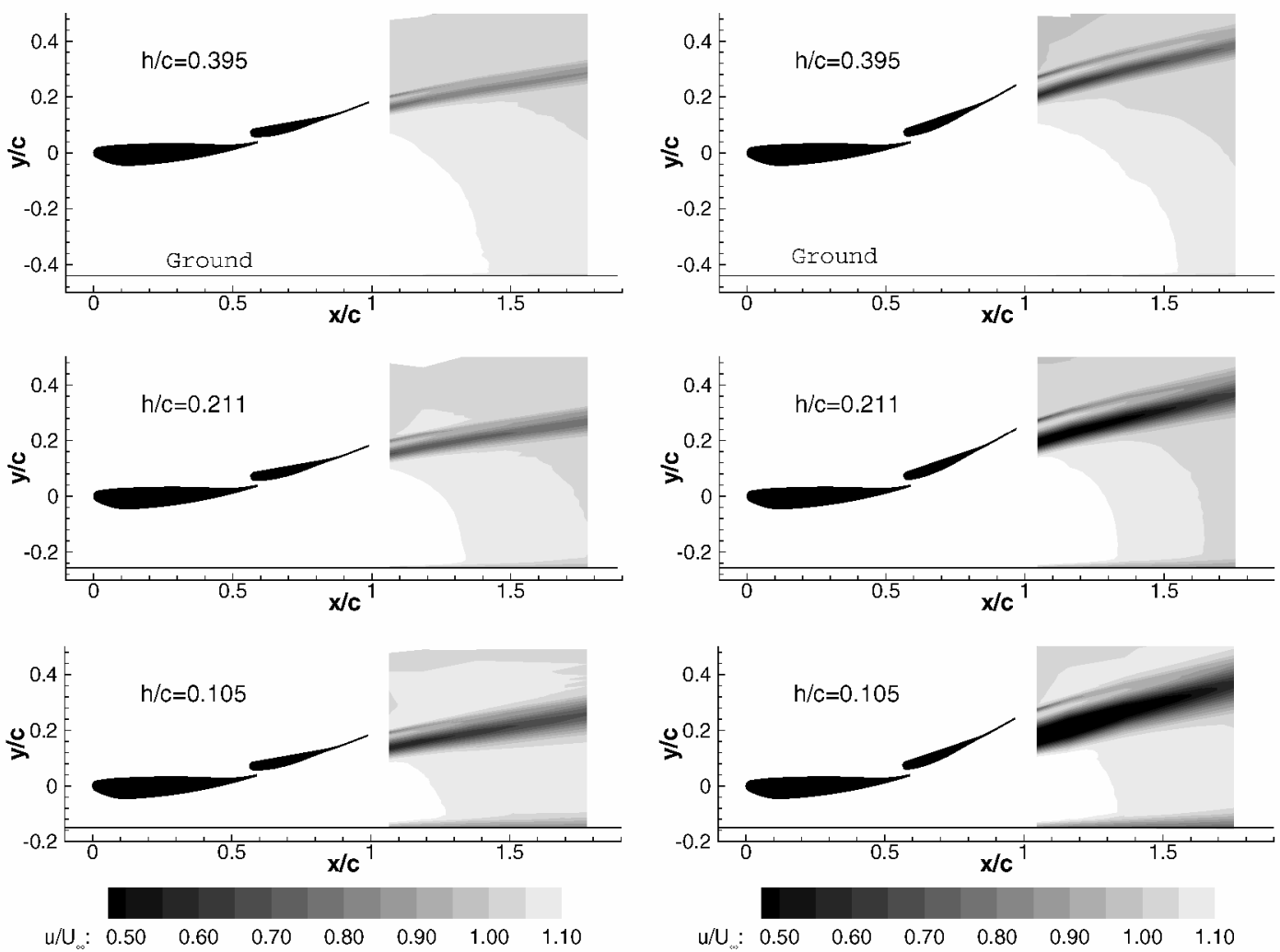

a) Low flap angle

b) High flap angle

Fig. 9 The $u / U_{\infty}$ contours.

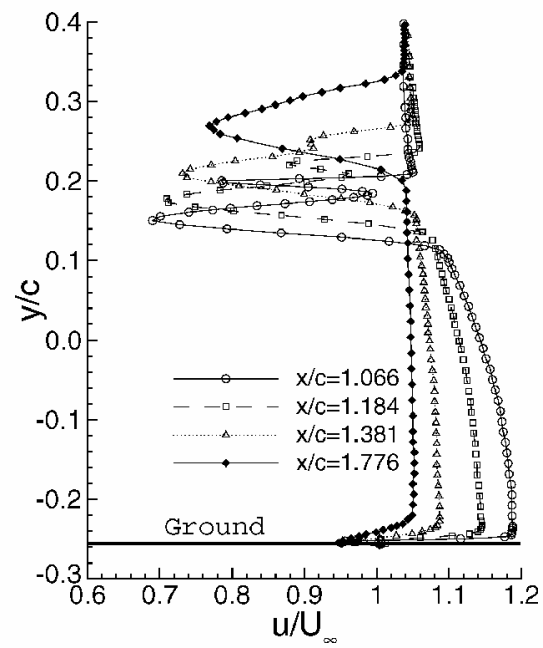

a) Streamwise velocity

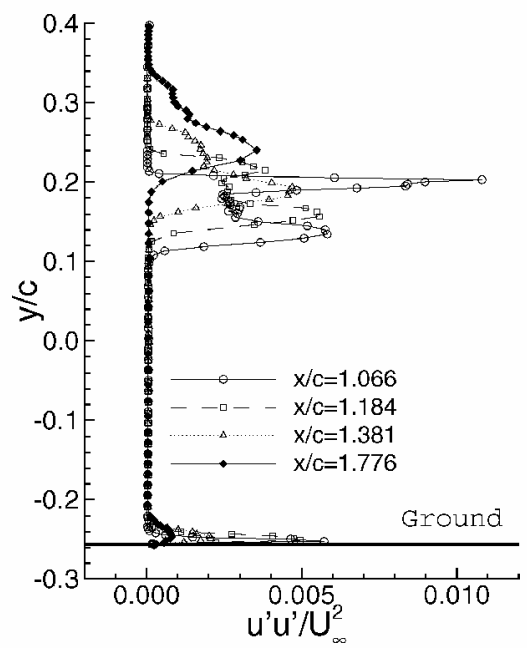

b) Normal stress

Fig. 10 Wake surveys for low flap angle at $h / c=0.211$.

\section{Off-Surface LDA Wake Survey}

An LDA wake survey was taken at four streamwise locations of $x / c=1.066,1.184,1.381$, and 1.776 behind the trailing edge of the flap. Boundary-layer surveys were taken along a line perpendicular to the suction surface, at the trailing edge of the flap (see Fig. 2). Results were acquired at heights of $h / c=0.395,0.211$, and 0.105 for the low and the high flap angles.

A complex turbulent wake flow is produced by the wing (Figs. 911). Immediately downstream of the low angle flap, two minima in $u$ were found (Figs. 9a and 10a); the more significant in terms of velocity deficit and thickness appears to be caused by the main element. As the wake develops downstream, turbulentmixing increases the size of the wake (Fig. 10b), as was found for the single-element wing, ${ }^{5}$ and the maximum velocity deficit reduces. The wake surveys show that the flap wake would mix with the wake from the main element, such that at $x / c=1.776$ for $h / c=0.211$; no sharp discontinuity in the profile exists, indicating that the wakes are fully merged at this location. At $x / c=1.066$ the flow velocity increases from the wake to the ground. This vertical pressure gradient is as would be expected from vertical traverses not perpendicular to the curved surface of the flap. Close to the trailing edge, the flow is at a higher velocity than freestream in the region between the wing and the ground. In the region from the wake to the ground, the adverse pressure gradient can be seen to reduce the velocities in the streamwise direction. The final point, at $x / c=1.776$, shows a relatively constant velocity profile in this region. The boundary layer very close to the ground can be seen and is more prominent than the single-element wing. For $h / c=0.211$ at the low flap angle, 


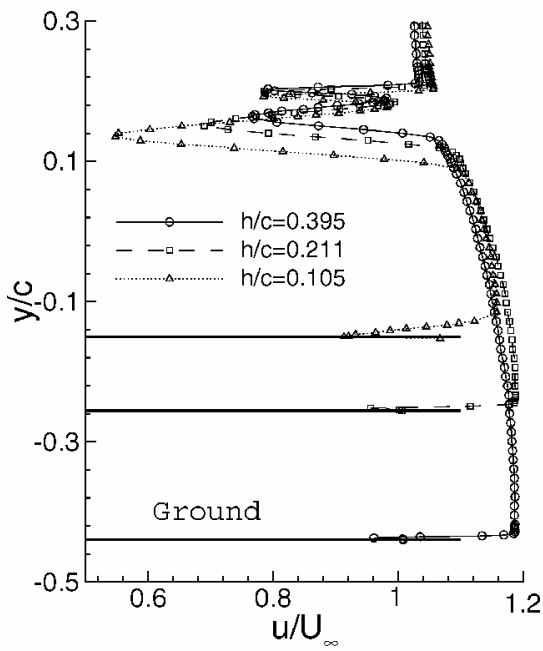

a) Different heights at low flap angle

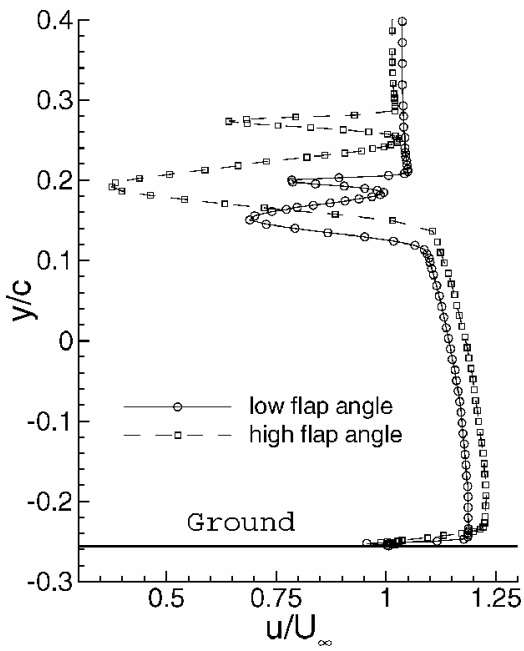

b) Effect of flap angle at $h / c=0.211$

Fig. 11 Wake surveys at $x / c=1.066$.

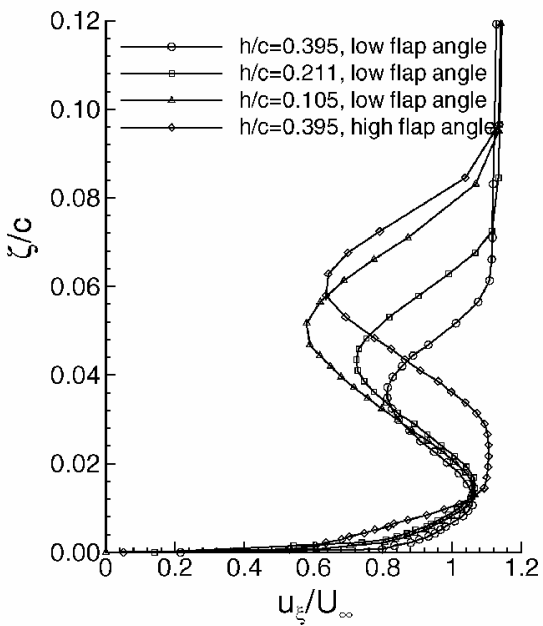

a) Tangential velocity

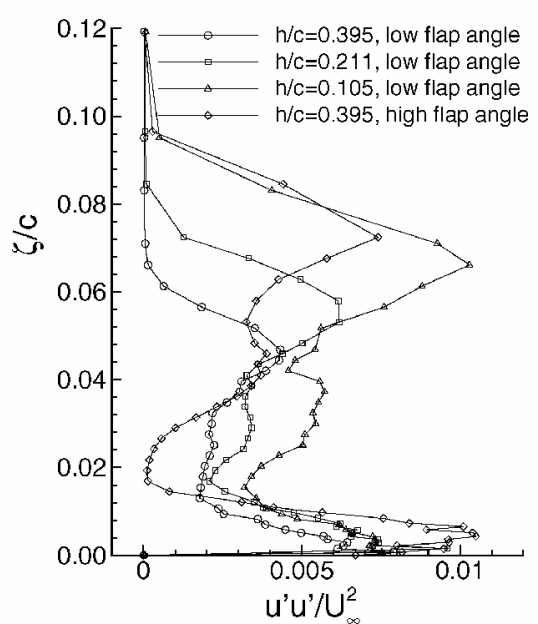

b) Normal stress

Fig. 12 Boundary layer at flap trailing edge.

the velocity deficit appears similar as the flow moves downstream, with a minimum velocity of $u / U_{\infty} \approx 0.95$. The layer does, however, appear to have grown in thickness.

The turbulent stress $u^{\prime} u^{\prime} / U_{\infty}^{2}$ distribution (Fig. 10b) shows two distinct peaks at $x / c=1.066$, representing the wakes from the flap and the main element. Here, the contribution from the flap is more significant than that of the main element. At the next point, $x / c=1.184$, the perturbations from the main element have reduced only slightly in the portion of the wake from the main element but significantly in the portion from the flap. At $x / c=1.381$ the flap contribution falls again and is more like a plateau, until at $x / c=1.776$ the results show advanced merging, with only a small bump. High levels of the perturbationvelocity, which decrease significantly with distance downstream, can be seen in the boundary layer close to the ground.

As the height of the wing is reduced (Fig. 11a), the wake from the wing increases as was found for the single-element wing. However, the portion of the wake from the flap does not change significantly, in terms of the velocity and the thickness. The portion from the main element is the cause of the increase; the wake thickens, and the velocities reduce as the height is reduced. In a similar manner to the single-element wing, it is the portion from the suction surface (of the main element) that changes, and the pressure surface contribution does not vary significantly. Similar values for the velocity are found in the region of accelerated fluid between the wake and the ground. The retarded flow very close to the ground becomes more significant as the height is reduced, as the layer becomes thicker, and possibly becomes more extreme in terms of the velocity deficit.

Both the velocity deficit and the wake thickness are greater for the high flap angle, not only because of the main element, but also from the flap (Figs. 9a and 11b). From the wake surveys it can be seen that, for the high flap angle, the wakes are further from merging than for the low flap angle. The flow between the wing and the ground is accelerated to a greater extent for the high flap angle for the results near to the wing. For $h / c=0.105$ the contours show that the velocity deficit very close to the ground is greater for the high flap angle, both in terms of the velocity and the thickness. At the greater heights it is clear that the layer thickens as it moves downstream, but it is difficult to compare the velocities directly. The general effect of changing the height on the flowfield and the development of the wake downstream is similar to those found for the low flap angle.

Boundary-layerprofiles (Fig. 12a) confirm that at the trailing edge of the flap the wake from the main element is indeed separated from the boundary layer of the flap and that the merging of the layers, if any has happened, is small at this streamwise location. As the height is varied for the low flap angle, the boundary layer directly from the flap changes little, and the results are within the positional accuracy of the equipment used. The velocity at the confluence between the layers is $u_{\xi} / U_{\infty} \approx 1.06$ for the three heights for the low flap angle. The minimum velocity caused by the wake from the main element reduces as the height reduces, and the location of this moves farther 

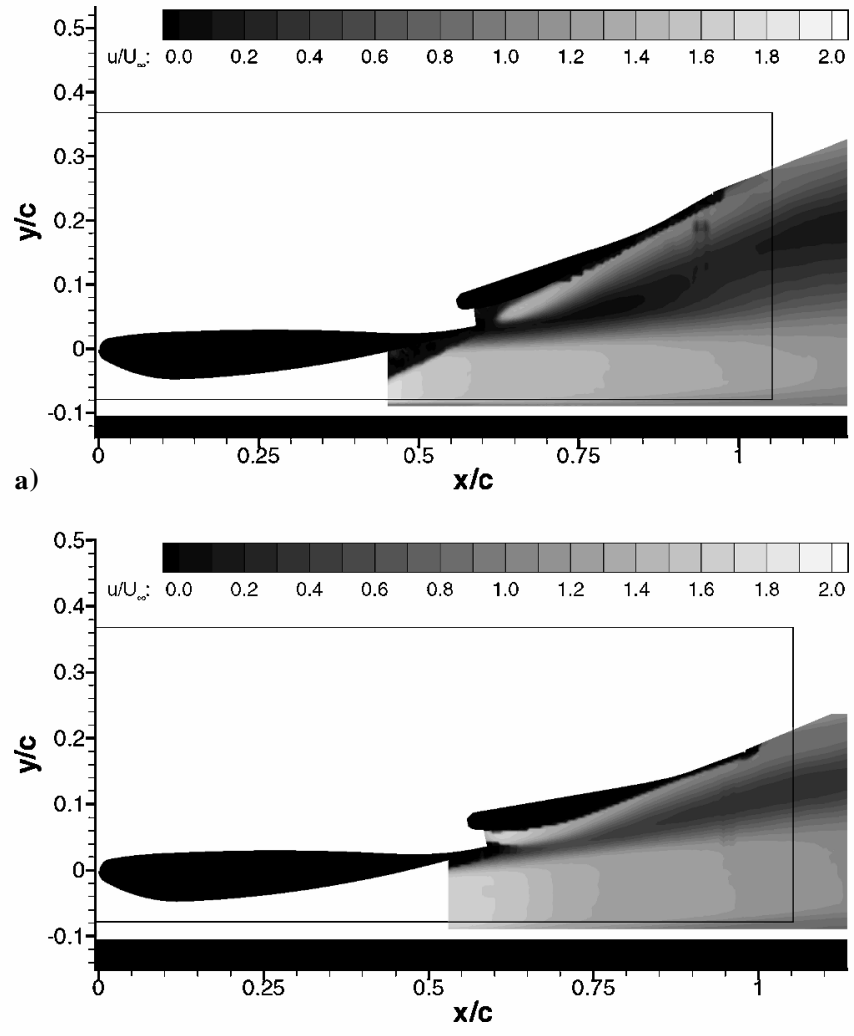

c)

Fig. 13 Off-surface PIV survey: $u / U_{\infty}$ contours; a) $h / c=0.058$, high flap angle, b) $h / c=0.132$, high flap angle, c) $h / c=0.058$, low flap angle, and d) $h / c=0.132$, low flap angle.

away from the surface, as the wake thickness increases. The effect of the overall boundary-layer thickness increasing as the ground is approached is caused by the contribution from the main element. For the high flap angle at $h / c=0.395$, the boundary layer directly from the flap is thicker. In addition, the merging of the wake from the main element with the flap boundary layer is less developed than for the results at the low flap angle. The main element boundary layer is both thicker and more significant in terms of the velocities, compared to the low flap angle.

The turbulent stress $u^{\prime} u^{\prime} / U_{\infty}^{2}$ is shown in Fig. 12b for the boundary-layer profiles. For the low flap angle concentrations in $u^{\prime} u^{\prime} / U_{\infty}^{2}$ are found in the region close to the flap surface. The highest values are found at values of $\zeta$ less than $0.002 c$ from the surface, and this decreases to minima at $\zeta \approx 0.015 c$, a height near the merging of the main-element wake and the flap boundary layer. In the region of the main-element wake, $u^{\prime} u^{\prime} / U_{\infty}^{2}$ increases to a maximum. For all three heights this maximum is at a location farther away than the center of the main-element wake, and the magnitude increases as the height is reduced. Some evidence of a second peak of perturbations exists at a location closer than the center of the boundary layer, but this is not well defined. The curve then drops as the edge of the boundary layer is approached. For the high flap angle the peak within the flap boundary layer is of a greater magnitude than the low flap angle at $h / c=0.395$. The perturbations then drop sharply to practically zero, confirming that this is out of, or very close to, the edge of the main-element wake. The curve increases to a first small peak, on the inner side of the center of the wake, drops slightly, and increases again to a second peak, farther out from the center of the wake, then falling off to the edge of the boundary layer.

\section{Off-Surface PIV Survey}

Although the LDA survey provided both qualitative and quantitative data of the wake development, it does not extend to the region underneath the flap and above the ground. Features in the region would provide insight into the force reduction at the low ground height. PIV surveys were therefore performed in the region between the flap and the ground. The survey was made feasible by the use of
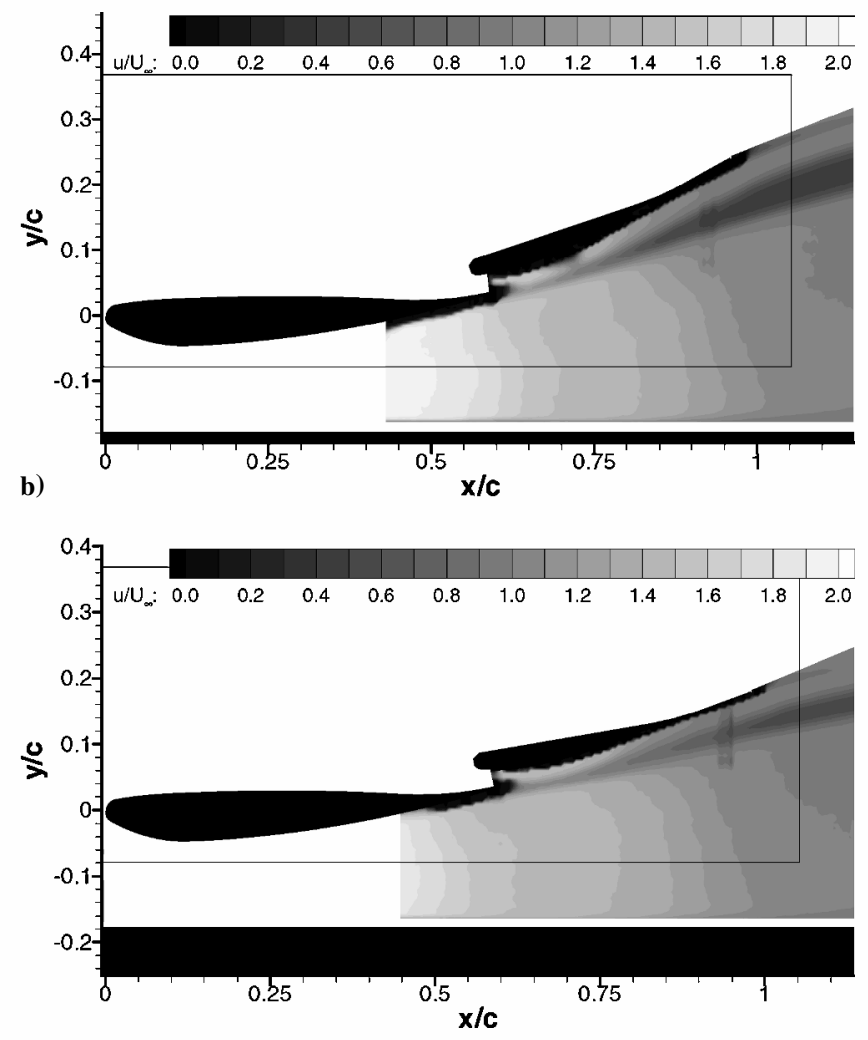

d)

glass end plate. Figure 13 gives mean streamwise velocity contours at two typical heights: one in the force reduction region and another in the enhancement region.

The PIV surveys generally confirm the observations made with the LDA surveys with additional new information. The wake from the main element is seen to dominate, and its thickness increases as the ground heightis reduced, as observedin the LDA measurements. The flow accelerates between the ground and the main element to reach a speed higher than the freestream before the maximum suction point (not shown in the PIV images); hence the high velocity region between the ground and the wing. After the maximum suction the wing effectively forms a diffuser section with the ground, and the flow decelerates downstream. For the majority of test cases, the flow stays attached at the trailing edge of the main element, unlike the single-element case. ${ }^{3}$ The Kutta condition at the trailing edge is satisfied, and the wake off the trailing edge of the main element follows an upward path (Fig. 13b). Nevertheless, the wakes from the main element and the flap do not merge immediately after the flap, and the main-element wake does not touch the flap for all cases.

For the high flap angle, at the low ground heights of $h / c=0.058$ and 0.066 , a different flow pattern emerges. The flow on the suction surface of the main element is separated at the trailing edge, although the extent of the separation is small. (See Fig. $8 \mathrm{~b}$ and note that the separation is not shown in the PIV images because of reflection.) Nevertheless, with the appearance of the separation, the Kutta condition is not satisfied at the trailing edge. As a result, the lower bound of the wake from the main element now experiences a downward trend. In fact, a wall-jet-like flow now exists between the ground and the wing. Inspection of the mean velocity distribution immediately points to the existence of a minimum point in the streamwise velocity in the wake from the main element. The position of the minimum, though, differs for the low ground heights and high ground heights. At $h / c=0.058$ and 0.066 the minimum is located immediately after the main element and beneath the flap, whereas for the higher ground heights for the high flap angle and for the low flap angle cases the minimum is located after the flap (see Table 1). 
Table 1 Measured minimum in streamwise velocity in the main-element wake

\begin{tabular}{lccccccc}
\hline \hline & \multicolumn{3}{c}{ High flap angle } & & \multicolumn{3}{c}{ Low flap angle } \\
\cline { 2 - 4 } \cline { 6 - 7 }$h / c$ & $u / U_{\infty}$ & $x / c$ & $y / c$ & & $u / U_{\infty}$ & $x / c$ & $y / c$ \\
\hline 0.058 & 0.04 & 0.80 & 0.06 & & 0.30 & 1.03 & 0.12 \\
0.066 & 0.16 & 0.82 & 0.08 & & 0.37 & 1.04 & 0.13 \\
0.071 & 0.04 & 1.06 & 0.17 & & 0.40 & 1.06 & 0.13 \\
0.079 & 0.08 & 1.07 & 0.18 & & 0.44 & 1.08 & 0.14 \\
0.084 & 0.14 & 1.08 & 0.19 & & 0.46 & 1.09 & 0.14 \\
0.092 & 0.20 & 1.09 & 0.20 & & 0.47 & 1.07 & 0.14 \\
0.097 & 0.25 & 1.08 & 0.19 & 0.48 & 1.10 & 0.15 \\
0.105 & 0.31 & 1.11 & 0.21 & 0.49 & 1.09 & 0.15 \\
0.118 & 0.37 & 1.12 & 0.21 & & 0.51 & 1.11 & 0.16 \\
0.132 & 0.41 & 1.10 & 0.21 & & 0.53 & 1.12 & 0.16 \\
0.158 & 0.44 & 1.12 & 0.22 & & 0.55 & 1.11 & 0.16 \\
0.184 & 0.47 & 1.14 & 0.23 & 0.64 & 1.12 & 0.16 \\
0.211 & 0.48 & 1.13 & 0.23 & 0.64 & 1.12 & 0.17 \\
\hline \hline
\end{tabular}

\section{Further Discussion}

Adding the flap to the main element induces a greater circulation around the main element, as can be seen by the greater suction on the lower surface and the increased pressures on the upper surface. The fact that there is a finite suction at the trailing edge of the main element implies that the pressure recovery from the suction peak on the main element to the trailing edge is not as severe. Large regions of separated flow were observed for the single-element wing, but for the double-element wing separation is not as widespread. The two effects, the circulation effect and the dumping effect, were described by $\mathrm{Smith}^{7}$ as contributing factors to the benefit of a multielement configuration.

For the double-element wing a force reduction region $c$ is identified, similar to the single-element wing. ${ }^{3}$ Above the region $c$ two regions of force behavior are identified: regions $a$ and $b$. Above region $a$ heights, at and above $h / c=0.171$ for the low flap angle and at and above $h / c=0.237$ for the high flap angle feature an increasing downforce with an increasing proximity to the ground. The slope increases as the height reduces. Below this, at region $b$ heights, the curve also increases with reducing ground height until the maximum downforce is obtained. For the low flap angle there is an asymptotic increase and then a smooth reduction in the gradient to the maximum downforce, followed by a reduction in downforce. For the high flap angle the curve is more linear, and there is a sharp reduction below the maximum downforce. At the boundary between the type $a / b$ flows, there is a discontinuity in slope in the downforce curve. For the low flap angle this is manifested as a plateau region at the large type $b$ heights. For the high flap angle there is a sudden reduction in downforce, compared to the lowest type $a$ heights.

At the center of the wing, the contribution from the flap at the low flap angle increases by about $30 \%$ as the height is reduced from $h / c=0.592$, until a maximum at $h / c=0.105$, and then reduces a little below this. Similar results are found for the high flap angle, where the height at the maximum sectional downforce is $h / c=0.158$. The pressure distributions suggest that the small reductions in downforce are caused by reductions in pressure on the pressure surface and small reductions in suction in the region $x / c=0.65-0.8$ on the flap suction surface. The general increase in downforce as the ground is approached for the flap is significantly smaller than that for main element. As the height of the wing is changed, the flap is farther from the ground and therefore less sensitive to changes in ground height than the main element. There is a more significant reduction in downforce at the lowest height for the high flap angle, which is caused by the boundary layer separating over the flap. The lower circulationimparted by the flap on the main element can be seen represented by the lower suction on the main element suction surface for the lowest height. According to LDA measurements, two hypotheses are presented. First, the boundary layer can separate from the main-element suction surface for the lowest height with the high flap angle, preventing the wake from flowing over the flap, leading to the flap boundary layer separating and a lower flap loading. However, the flap flow can separate itself, causing a loss in flap circulation, reducing the main-element circulation. The PIV measurements suggest that the first is the case. The existence of the trailing-edge separation leads to a downward movement of the lower bound of the wake from the main element and the presence of a velocity minimum in the wake immediately after the main element and beneath the flap. This creates an effective diffuser section between the wake and the flap and places a higher pressure recovery demand on the flap suction surface flow, leading to separation and downforce loss.

\section{Summary}

The aerodynamic behavior of a cambered, double-element, highlift wing has been studied using model tests. Techniques employed include force balance, surface oil flow visualization, surfacepressuretaps, off-surfaceLDA surveys, and PIV measurements. The effects of ground proximity and flap angle deflection are quantified.

It was found that the main element produces most of the downforce and dominates the turbulent wake development. The ground proximity does not alter the turbulent wake from the flap significantly, in terms of the velocity deficit and the thickness. An increase in the wake thickness and a reduction in the velocity deficit are found in the portion from the suction surface of the main element, as the wing is moved to the ground. Both the velocity deficit and the wake thickness are greater for the high flap angle, not only because of the main element, but also from the flap. In the center of the wing, the flow can be regarded as quasi-two-dimensional. Three regions are identified on the downforce with height curve. A force reduction region $c$, similar to that of a single element wing, is presented for the two flap settings. Above the force reduction region $c$, there are two distinct regions. At large heights, region $a$, the downforce increases asymptotically with a reduction in height. Then there is either a small plateau, in the case of the low flap angle, or a reduction in downforce, in the case of the large flap angle. The downforce then increases again, region $b$, until it reaches a maximum, and then reduces. In the case of the low flap angle, the maximum downforce is dictated by gains in downforce from lower surface suction increases and losses in downforce caused by upper surface-pressure losses and lower surface suction losses, with a reduction in height. For the high flap angle there is a sharp reduction just beyond the maximum because of the boundary layer separating and a resultant loss of circulation on the main element.

\section{Acknowledgments}

J. Zerihan is supported by an Engineering and Physical Sciences Research Council studentship. The authors thank BAR for support and $\mathrm{W}$. Toet for discussions during the course of the study and J. McManus and S. Mahon for performing the PIV survey.

\section{References}

${ }^{1}$ Agathangelou, B., and Gascoyne, M., "Aerodynamic Considerations of a Formula 1 Racing Car," Society of Automotive Engineers, Publ. 980399 Feb. 1998

${ }^{2}$ Jeffrey, D., and Alperin, M., "Aspects of the Aerodynamics of Year 2000 Formula One Racing Cars," 3rd MIRA International Vehicle Aerodynamics Conference, MIRA, 2000

${ }^{3}$ Zerihan, J., and Zhang, X., "Aerodynamics of a Single-Element Wingin-Ground Effect," Journal of Aircraft, Vol. 37, No. 6, 2000, pp. 1058-1064

${ }^{4}$ Knowles, K., Donahue, D. T., and Finnis, M. V., "A Study of Wings in Ground Effect," Loughborough University Conference on Vehicle Aerodynamics, Royal Aeronautical Society, London, 1994, pp. 22.1-22.13.

${ }^{5}$ Zerihan, J., and Zhang, X., "Unsteady Turbulent Wake Behind a SingleElement Wing in Ground Effect," 10th International Symposium on Applications of Laser Techniques to Fluid Mechanics, Lisbon, July 2000 , Paper 8-1.

${ }^{6}$ Zerihan, J., and Zhang, X., "A Single Element Wing in Ground Effect: Comparisons of Experiments and Computations," AIAA Paper 2001-0423, Jan. 2001.

${ }^{7}$ Smith, A. M. O., "High-Lift Aerodynamics," Journal of Aircraft, Vol. 12, No. 6, 1975, pp. 501-530.

${ }^{8}$ Ranzenbach, R., Barlow, J. B., and Diaz, R. H., "Multi-Element Airfoil in Ground Effect-An Experimental and Computational Study," AIAA Paper 97-2238, June 1997 
${ }^{9}$ Jasinski, W. J., and Selig, M. S., "Experimental Study of Open-Wheel Race-Car Front Wings," Society of Automotive Engineers, Publ. 983042, Nov. 1998.

${ }^{10}$ Zerihan, J., "An Investigation into the Aerodynamics of Wings in Ground Effect," Ph.D. Dissertation, Aeronautics and Astronautics, School of Engineering Sciences, Univ. of Southampton, Southampton, England, U.K., April 2001.

${ }^{11}$ Moffat, R. J., "Contributions to the Theory of Single-Sample Uncertainty Analysis," Journal of Fluids Engineering, Vol. 104, June 1982, pp. 250-260.

${ }^{12}$ Zhang, X., "An Inclined Rectangular Jet in a Turbulent Boundary Layer-Vortex Flow," Experiments in Fluids, Vol. 28, 2000, pp. 344-354.

${ }^{13}$ Benedict, L. H., and Gould, R. D., "Towards Better Uncertainty Estimates for Turbulence Statistics," Experiments in Fluids, Vol. 22, No. 2, 1996, pp. 129-136.

${ }^{14}$ Zhang, X., Zerihan, J., Ruhrmann, A., and Deviese, M., "Tip Vortices Generated by a Single-Element Wing in Ground Effect," 11 th International Symposium on Applications of Laser Techniques to Fluid Mechanics, Lisbon, July 2002, Paper 4-1.

A. Plotkin Associate Editor 